\title{
OPORTUNIDADES PARA LA TRANSFORMACIÓN DIGITAL DE LA CADENA DE SUMINISTRO DEL SECTOR BANANERO BASADO EN SOFTWARE CON INTELIGENCIA ARTIFICIAL
}

\begin{abstract}
Isabel Cristina Arango Palacio
Magister Logística Integral, Docente, Politécnico Colombiano Jaime Isaza Cadavid, isabelarango@elpoli.edu.co.

RESUMEN

La inteligencia artificial ofrece grandes oportunidades para la cadena de suministro, siendo esto una ventaja competitiva para el mercado cambiante de hoy en día. Este artículo tiene como objetivo identificar los impactos y oportunidades que puede ofrecer el software con inteligencia artificial para facilitar la operación y mejorar el desempeño de la cadena de suministro en el sector bananero de Colombia. La metodología de trabajo consta de seis pasos en donde se obtuvo un total de 72 investigaciones. Las fuentes de información fueron cuatro bases de datos. Como conclusión principal, la cadena de suministro del sector bananero tiene todo lo necesario para que se implementen soluciones basadas en software inteligente con el fin de lograr una adaptación, flexibilidad y sensibilidad al contexto y dominio de ejecución.
\end{abstract}

Palabras clave: Software; inteligencia artificial; sector bananero; cadena de suministro; transformación digital.

Recibido: 15 de enero de 2021. Aceptado: 25 de abril de 2021

Received: January 15, 2021. Accepted: April 25, 2021

DOI: http://dx.doi.org/ 10.33571/rpolitec.v17n33a4

\section{OPPORTUNITIES FOR THE DIGITAL TRANSFORMATION OF THE SUPPLY CHAIN OF THE BANANA SECTOR BASED ON ARTIFICIAL INTELLIGENCE SOFTWARE}

\begin{abstract}
Artificial intelligence offers great opportunities for the supply chain, making it a competitive advantage for today's changing market. This paper aims to identify the impacts and opportunities that artificial intelligence software can offer to supply chain in the Colombian banana sector to facilitate the operation and improve the performance. The searching method consists of six steps getting 72 investigations finally. The sources of information were four databases. The main conclusion is the supply chain of the banana sector has everything for implementation of solutions based on intelligent software in order to achieve adaptation, flexibility and context awarenes and execution domain.
\end{abstract}

Keywords: software, artificial intelligent, banana sector, supply chain, digital transformation..

Cómo citar este artículo: I.C. Arango-Palacio. "Oportunidades para la transformación digital de la cadena de suministro del sector bananero basado en software con inteligencia artificial", Revista Politécnica, vol. 17, no.33 pp.47-63, 2021. DOI: http://dx.doi.org/ 10.33571/rpolitec.v17n33a4 


\section{INTRODUCCIÓN}

La gestión de la cadena de suministro es clave para el éxito de las empresas y para la economía en general; tener precisión en la toma de decisiones ofrece una ventaja competitiva importante, sin embargo, existen muchos factores internos y externos que afectan el desempeño de la cadena de suministro [1]. El sector agrícola y es especial el bananero, es significativo para la economía nacional y no está exento de los factores internos y externos que limitan el desempeño de su cadena de suministro [2]. En los últimos años se ha demostrado que la Inteligencia Artificial (IA), apoya la capacidad humana, permitiendo una mejora que no se había considerado posible, ayudando así a la humanidad a potencializar las estrategias y creatividad en las empresas para lograr un crecimiento económico significativo donde es aplicada.

Nasiri et al. en [3] plantean que la cadena de suministro y su transformación digital, puede ser impactada de manera positiva con el uso de las técnicas de IA en sus soluciones, es aspectos tales como: integración negocios, reducción de costos, optimización de tiempos, recursos, entre muchos usos permitiendo visualizar cómo deben ser transformadas digitalmente las cadenas de suministro. Integrar la cadena de suministro con técnicas de la IA, vislumbra oportunidades para el sector bananero de manera. Así mismo, se debe tener en cuenta que la cadena de suministro depende de los detalles propios asociados a los productos que controla.

Debido a la importancia de dichas áreas, se han realizado trabajos de revisión sistemática del uso de técnicas de IA en las cadenas de suministro.

Wu et al., en [4] definen tendencias en investigación, que aún hoy en día siguen teniendo gran potencial del uso en la cadena de suministro del banano. Dichas tendencias se orientan a: implementación de análisis inteligente a partir de grandes volúmenes de datos, sistemas de software adaptativos al contexto y entorno, automatización de tareas y lógica de decisión e integración de información. Por su parte Zhao, Ji \& Feng en [5] concluyen retos para la cadena de suministro generalizada, tales como: Reducción de costos, gestión de los riesgos, fiabilidad de clientes, adaptación a la globalización y visibilidad.

El presente artículo propone una revisión amplia de las técnicas de IA y sus aplicaciones en la cadena de suministro del sector bananero, con el fin de identificar oportunidades y mejoras dentro de los procesos asociados, así como en los mecanismos de razonamiento, adaptación y reacción.

También se presenta en esta artículo, la metodología de trabajo empleada para realizar la revisión sistemática de artículos de interés, la cual consta de seis pasos a seguir y en cada uno de ellos se busca garantizar un orden y control para lograr resultados confiables que validen las posibles respuestas a preguntas como ¿Cuáles son los principales impactos que genera el software con inteligencia artificial en la cadena de suministro del sector bananero en Colombia? y ¿el software basado en inteligencia artificial brinda oportunidades de transformación digital a la cadena de suministro del sector bananero en Colombia?

Las condiciones de búsqueda están enfocadas en los siguientes filtros: títulos, resúmenes y palabras clave, en un intervalo de tiempo de cinco años comprendidos entre el 2015 y 2020, en artículos de contenido abierto y en los idiomas español e inglés. A partir de ello, se realizó una recopilación de los aportes de los autores a la gestión de la cadena de suministro en la transformación de la cadena de suministro.

El resto del artículo se estructura de la siguiente manera: en la sección 2 se presenta el marco conceptual que soporta la revisión, luego en la sección 3 la metodología de trabajo definida y los hallazgos de la revisión. La sección 4, presenta el análisis de los impactos de las técnicas de la IA respecto a las cadenas de suministro, y finalmente en la sección 6 se consolida la información de la transformación digital frente a los impactos analizados. 


\section{MARCO CONCEPTUAL}

Esta sección presenta los conceptos relacionados con el tema propuesto, donde el lector se contextualiza en; el sector bananero en Colombia, cadena de suministro inteligente, inteligencia artificial, sistemas expertos, lógica difusa, algoritmos genéticos, redes neuronales, agentes y software inteligente y transformación digital de la cadena de suministro.

\subsection{Sector bananero en Colombia}

El sector bananero en Colombia es significativo para la economía nacional y regional, la principal zona de producción y comercialización se encuentra en el Urabá antioqueño uno de los núcleos productivos más importantes está en los municipios Apartadó, Turbo y Chigorodó con una producción de 1.246.209 Ton.

En el 2018 se produjo en Colombia cerca de 100.491 .531 cajas de banano, 2.472738 más que en el 2017, el principal consumidor es la Unión Europea quien recibe el $82 \%$ de las exportaciones y Estados Unidos el $13 \%$ el sector bananero genera el 1,5\% del PIB agrícola $[6,7]$.

La cadena de suministro de las principales empresas bananeras está compuesta por proveedores, granjas, transportadores, distribuidores, grandes superficies y consumidores finales, en estas interrelaciones hay muchos desafíos asociados a la administración de la cadena de suministro, una de ellas es la necesidad de tener confianza entre las partes involucradas, mejorar la velocidad en la comunicación, crear vínculos de confianza, contar con transacciones de transparencia y tener trazabilidad en todas las operaciones necesarias para llegar a usuario final.

\subsection{Cadenas de suministro inteligente}

La cadena de suministro tradicional se caracteriza por flujos físicos que mueven productos, dinero e información entre todos los involucrados, desde la materia prima hasta la entrega a satisfacción de las solicitudes de un consumidor [8].

El concepto de cadena de suministro inteligente varía y aun no se encuentra un consenso académico sobre la definición, como por ejemplo en [5] proponen que la cadena de suministro inteligente es un sistema integrado construido dentro y entre empresas para combinar sistemas de gestión y tecnologías de información modernas para la intelectualización, digitalización, redes y automatización de la cadena de suministro.

Una cadena de suministro inteligente gestiona sus propios con una amplia variedad de tecnologías innovadoras, por ejemplo vehículos aéreos no tripulados, computación en la nube e internet de cosas, entre otros [6].

\subsection{Inteligencia artificial}

La inteligencia artificial (IA), es el área de estudio que hace uso de modelos computacionales para lograr que las máquinas realicen tareas que por ahora son realizadas mejor por los seres humanos [7]. Dichas tareas se orientan principalmente a resolver problemas propios de: el razonamiento, la percepción, la comunicación, el aprendizaje, la optimización, la actuación sobre entornos, la visión artificial, la planeación y la toma decisiones.

Una técnica de IA es vista como un método que utiliza conocimiento representado de tal forma que representa generalizaciones, es comprensible, modificable y usable [8]. Las técnicas de IA se han utilizado de acuerdo a la naturaleza del problema, es decir, aquellas diseñadas para el aprendizaje deben ser empleadas para problemas con características similares.

Las técnicas de IA más conocidas son: lógica difusa, sistemas expertos basados en reglas, algoritmos genéticos, redes neuronales artificiales, agentes inteligentes, visión artificial, planificación, entre otras. No obstante, las técnicas pueden ser combinadas, a lo que se conoce como técnicas híbridas [9]. La clasificación de las técnicas tiene un abanico mucho más amplio, para efectos de este artículo, se consideran aquellas técnicas de mayor relevancia identificadas en el estudio de la problemática, sin 

embargo, existen otras técnicas como: visión artificial, planificación, procesamiento de lenguaje natural, redes inteligentes de datos, entre otras.

Los sistemas expertos (SE) son sistemas basados en reglas de producción, que permiten un razonamiento similar al de un ser humano [10]. Se caracteriza por tener un alto rendimiento, confiabilidad y un rápido tiempo de respuesta; sin embargo, su debilidad es la complejidad en su mantenimiento, ya que los cambios en su base de conocimiento no es posible realizarlos de manera dinámica. Los sistemas expertos son una herramienta útil en el área de la ingeniería de conocimiento, precisamente ya que se orientan al diseño y construcción de reglas y bases de conocimiento que facilitan el razonamiento inteligente. Así mismo, son apropiados para complementarse con otras técnicas como las redes neuronales.

La lógica difusa (LD) es una técnica asociada al razonamiento aproximado, en donde un elemento puede pertenecer a uno o varios conjuntos en distinto nivel de pertenencia. La LD permite construir conceptos "vagos" y por tanto facilita la construcción de sistemas que razonan de manera distinta a la lógica booleana [11]. La LD, al igual que los SE son técnicas maduras que ya se han llevado a electrodomésticos, como la lavadora, aires acondicionados y a juguetes infantiles.

Los algoritmos genéticos (AG) es una técnica asociada a los problemas de automatización, los cuales buscan mejorar las soluciones a partir de la maximización de funciones objetivo [12]. Los AG pertenecen al área de la computación evolutiva, que se orienta al diseño de algoritmos que aprovechan las teorías de evolución, como lo son la selección natural, la reproducción y la mutación de individuos dentro de una población.

Dentro de las técnicas de aprendizaje de máquina, se encuentran las redes neuronales artificiales, las máquinas de soporte vectorial, el agrupamiento, entre otras [13]. Estas técnicas se caracterizan por trabajar con conjuntos de datos, para poder predecir, clasificar, agrupar o compararlos. A partir de las técnicas de aprendizaje se pueden implementar sistemas de recomendación o predicción, apropiados para distintos dominios.

Los agentes inteligentes, son técnicas propias de los ambientes distribuidos, considerados como la principal técnica dentro de la inteligencia artificial distribuida. Se conocen como componentes de software capaces de realizar tareas autónomas en un ambiente con el fin de lograr un objetivo [14]. Los agentes trabajan en conjunto con otros agentes y conforman lo que se conoce como sistemas multiagente (SMA), en donde se potencia la capacidad de comunicación, percepción, coordinación y negociación.

Las técnicas de IA descritas no representan la totalidad de ellas, pues existen un número mayor asociados a problemas específicos, como lo son: la visión artificial, procesamiento de lenguaje natural o la planificación. Cabe señalar que las técnicas seleccionadas es este artículo, son aquellas que se identificaron con moda dentro del estudio y análisis de la problemática.

\subsection{Transformación digital en cadenas de suministro}

La integración sistémica global de la cadena de suministro identifica cuatro requisitos de transformación para los ecosistemas de negocios digitales, que constituyen una base para el desarrollo de negocios e innovación [15] y los cuales se adoptan requisitos de transformación digital basada en la tecnología disruptiva para la cadena de suministro como es la inteligencia artificial, sistemas expertos, lógica difusa, algoritmos genéticos, redes neuronales, agentes y software inteligente.

La transformación digital dentro de las cadenas de suministro, busca potenciar los sistemas tecnológicos que la soportan. Ya sea los costos de producción, el gasto de maquinaria y materiales o la gestión de los datos. A esto se suma que es posible integrar sistemas ciber-físicos que permitan interacción con los ambientes y así de esta manera apuntarle a lograr una agricultura sustentable [16].

El uso de tecnologías de forma limpia sin mayor impacto sobre el ambiente, son una característica de la transformación digital dentro de las cadenas. Es así, que la agricultura 4.0, surge con la necesidad de fortalecer y adaptar los fundamentos de la industria 4.0 en las cadenas productivas de la agricultura [17]. 
La transformación digital en la cadena de suministro es un área abierta en investigación, debido a la capacidad de acoplarse con otras tendencias tecnológicas. A modo de ejemplo, trabajos como el de Ehie, I., \& Ferreira, L. M. D. [8], muestran cómo influye la digitalización de los procesos de la cadena de suministro en las capacidades y desempeño operacional, enfocados en la construcción de marcos de trabajo dentro del área de la transformación, para planear, diseñar, construir y distribuir productos dentro de la cadena de suministro.

\section{METOdOLOGÍA DE TRABAJO}

La metodología consta de seis pasos y en cada uno de ellos se busca garantizar un orden y control para lograr resultados confiables que validen la discusión propuesta. En la Figura 1, se observan los pasos propuestos; y en las siguientes subsecciones se describe el trabajo realizado.

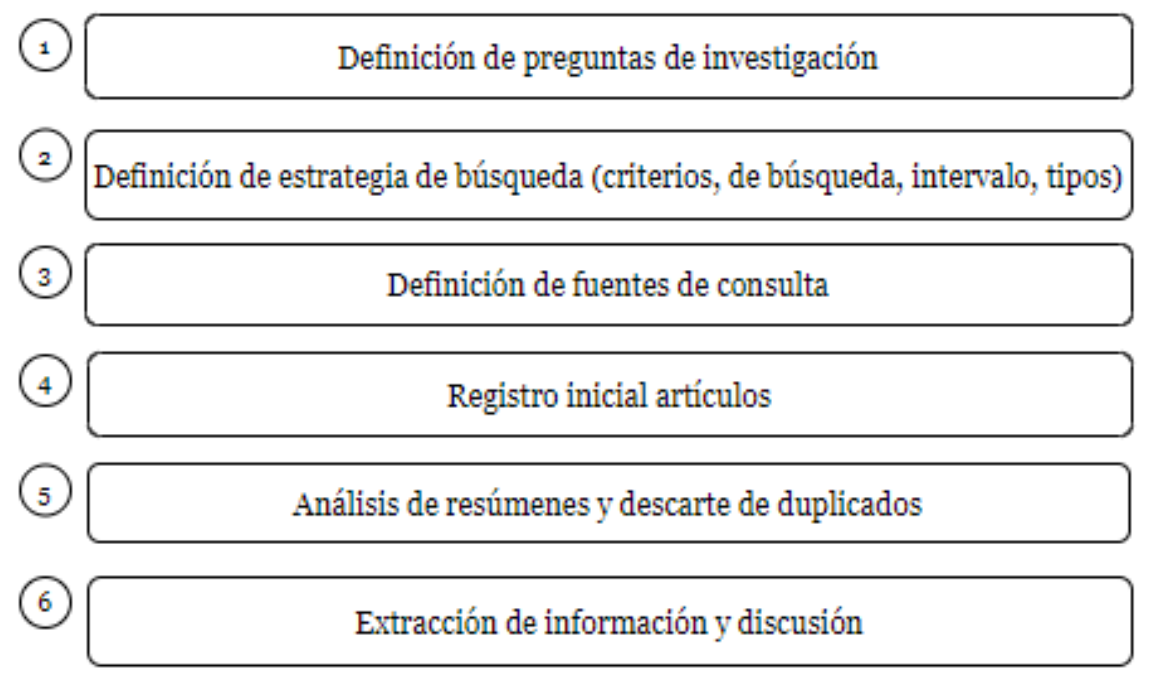

Figura 1 - Metodología de revisión de artículos

\subsection{Definición de preguntas de investigación}

La recopilación de la información se diseñó a partir de las siguientes preguntas de investigación:

- Pregunta 1: ¿Cuáles son los principales impactos que genera el software con inteligencia artificial en la cadena de suministro del sector bananero de Colombia?

- Pregunta 2: ¿El software con inteligencia artificial fortalece la cadena de suministro del sector bananero de Colombia frente a cambio críticos en el entorno?

\subsection{Definición estrategia de búsqueda}

Para realizar la búsqueda se definieron las siguientes condiciones:

- Los campos de búsqueda utilizados fueron: Título, resumen y palabras clave.

- El intervalo de tiempo fue de 5 años, es decir artículos comprendidos entre el 2015 al 2020.

- El tipo de artículos fue de contenido abierto (open access)

- Solo se consultaron artículos completos de revistas y memorias de congresos.

- Los criterios de búsqueda están relacionados de la siguiente forma: "Digital Supply chain AND Intelligent Software", "Supply Chain AND Artificial Intelligence" , "Supply chain AND genetic algorithm", "Supply chain AND intelligent agents" y "Supply chain AND Neural Networks".

- Las bases de datos seleccionadas fueron: Science Direct, Scopus, IEEE Explore, Proquest.

\section{REPORTE DE HALLAZGOS}

Una vez aplicada la estrategia de búsqueda sobre las fuentes definidas, se encontraron un total de 1530 artículos. A partir de este registro inicial se seleccionaron para un posterior análisis a 103 artículos. 
Tabla 1 - Consolidado registro inicial

\begin{tabular}{|l|c|c|c|c|}
\hline Criterio de búsqueda & Hallados & Seleccionados & Inglés & Citas \\
\hline Digital Supply chain AND Intelligent Software & 317 & 20 & 20 & 333 \\
\hline Supply Chain AND Artificial Intelligence & 148 & 14 & 14 & 281 \\
\hline Supply Chain AND genetic algorithm & 501 & 47 & 47 & 483 \\
\hline Supply Chain and Neural Networks & 391 & 5 & 5 & 30 \\
\hline supply chain and intelligent agents & 163 & 7 & 7 & 28 \\
\hline Total consultados & 1530 & 103 & & 1125 \\
\hline Filtro final & & 72 & & \\
\hline
\end{tabular}

Así mismo se puede observar que los algoritmos genéticos es una de las técnicas que presenta un mayor número de aplicaciones. Este fenómeno puede darse, debido a que las cadenas de suministro son susceptibles a mejoras por medio de técnicas de optimización, ya sea para reducir costos o incrementar la producción.

Una vez se hizo una lectura de resúmenes y eliminación de artículos duplicados que se encontraron en uno o varios criterios, se concretaron un total de 72 artículos, los cuales se emplearon para hacer la extracción de información y la correspondiente discusión. Con base en lo anterior, en el Figura 2 se presenta el comportamiento por años de las publicaciones seleccionadas. Se observa un crecimiento en los últimos 2 años, que se supone se debe al auge de las tecnologías de la industria 4.0 y su posible aplicación en la cadena de suministro. En total, en el periodo de 2018 y 2019 se tiene aproximadamente un $48 \%$ del total de los artículos.

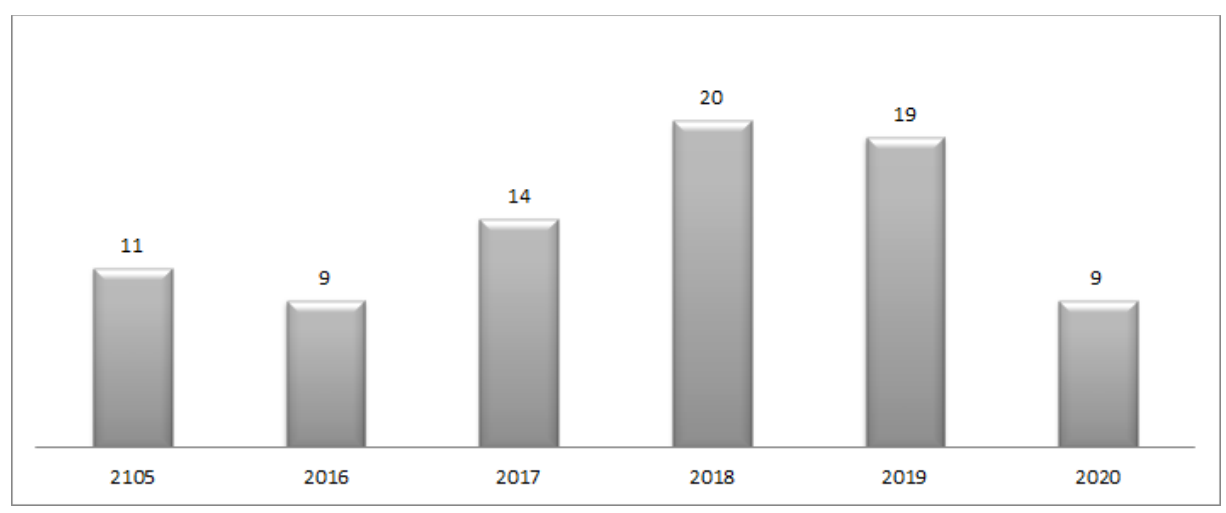

Figura 2 - Artículos distribuidos por años

En la Figura 3, se observa la relevancia de las soluciones desde el punto de vista de la sumatoria de citaciones de los artículos en cada uno de los criterios de búsqueda empleado. Nuevamente los AG presentan un dato significativo, debido a que las tecnologías y soluciones dentro de la cadena de suministro, por ahora solo se enfoca a mejorar tiempos y gastos. 


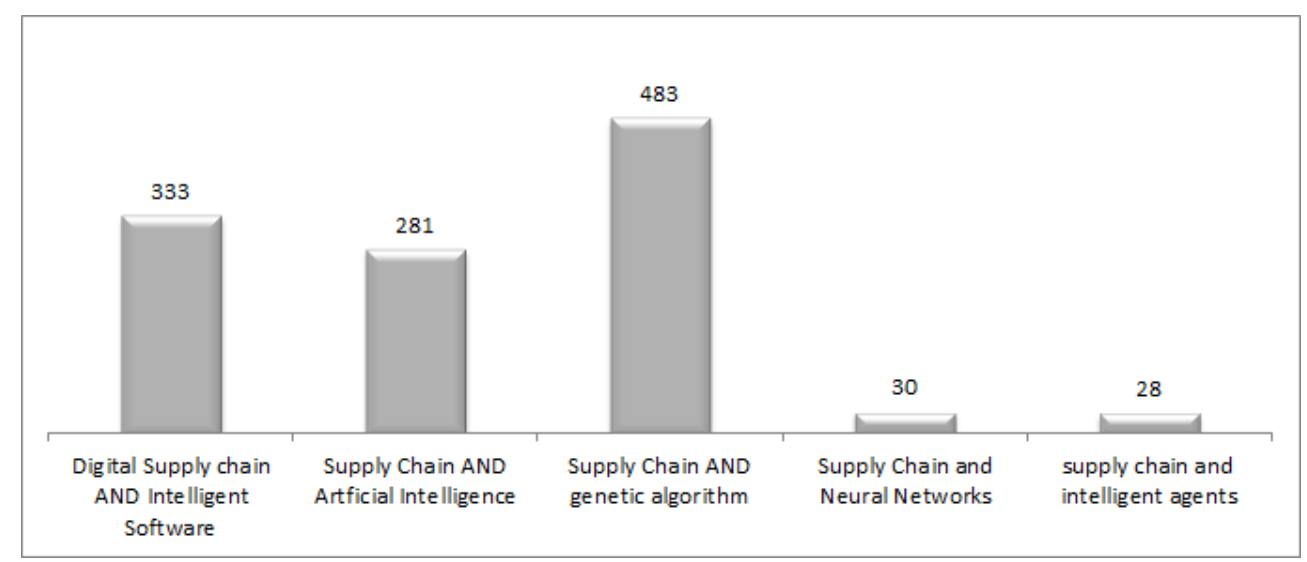

Figura 3 - Citaciones por tema

\subsection{Descripción de los impactos de la IA}

Esta sección presenta el análisis de los artículos seleccionados, se discuten los impactos de cada una de las técnicas con su aplicación en soluciones propias de la cadena de suministro, mencionando las áreas en las cuales aporta cada técnica de IA mencionadas previamente.

\subsection{Algoritmos genéticos}

Una de las principales áreas en la cuales impactan los AG en la cadena de suministro es la optimización en la producción y en el uso de recursos; En [18] propone una solución basada en AG que permite optimizar los niveles de producción en cadena de ciclos múltiples. Igualmente en [19] y [20] plantean modelos en red para mejorar el rendimiento en la producción de múltiples productos.

De manera similar, hay investigaciones orientadas a reducir la generación de materiales de desecho en las cadenas de suministro, ya sea en el sector de alimentos [21] o el sector de la construcción [22]. Así mismo las investigaciones se enfocan en mejorar la sostenibilidad de las cadenas, como por ejemplo, midiendo y reduciendo la huella de carbono generada [23]. Otros trabajos se han enfocado en la optimización de la producción, en problemas específicos como la programación y asignación de turnos de despacho en muelles [24], en sistemas de planeación en cargas [25] y en mejoramiento de mecanismos de distribución en sistemas de bodegas [26].

En las cadenas de suministro se viene trabajando fuertemente con los AG en el área de problemas de ruteo; tal es el caso de las investigaciones [27,28,29] que proponen sistemas de optimización de rutas internas para la ubicación y carga de los productos en almacenados en bodegas, permitiendo utilizar distintos tipos de vehículos de acuerdo al contexto, que conllevan a la reducción de costos y mejoramiento del servicio. Así mismo se han hecho propuestas para mejorar la entrega y recogida de productos, optimizando la asignación de vehículos respeto al lugar de residencia de los clientes $[30,31,32]$ y la programación de los despachos de acuerdo a las órdenes de pedido $[33,34,35]$.

Otra área representativa de impacto de los $A G$ es el diseño y modelamiento de las cadenas de suministro. Por ejemplo, en la minimización de costos [36], el diseño de secuencias de producción en ambientes de circuitos cerrados [37-40] y diseño de flujos con intervalos variantes [41]. Un enfoque con gran potencial dentro del diseño y modelamiento es la construcción de redes de integración entre las cadenas de suministro $[42,43,44]$.

Por último, y no menos importante se presentan aportes de los $A G$ en el área de almacenamiento y gestión de inventarios [45-51] y en el área de selección, evaluación de proveedores respecto a una demanda variable [52-57]. 


\subsection{Agentes inteligentes}

Teniendo en cuenta que los agentes inteligentes son componentes de software que permiten la percepción y actuación sobre un entorno, así como la coordinación, colaboración, negociación e integración de información cuando se construyen sistemas basados en agentes, se presentan aportes interesantes en la cadena de suministro.

En [58] proponen un sistema de percepción y razonamiento sobre una cadena de suministro que puede ser atendida por un agente y su percepción; así mismo, En [12] proponen una aproximación basada en agentes para la distribución y razonamiento de funciones.

Es importante señalar que otra fortaleza de los agentes, es la capacidad de estructurar sistemas multiagente (SMA), en donde se afianza la coordinación y negociación. En [59-61] presentan soluciones basadas en SMA donde es posible negociar sobre políticas para complementar los procesos de producción y logística; así mismo, permiten que dicho intercambio genere datos de aprendizaje con lo cual sea posible implementar métodos de predicción. Así mismo, los SMA fomentan la cooperación entre los agentes con el fin de lograr objetivos en común [62]. A esto se suma, que los SMA por su capacidad de interacción, percepción y actuación sobre el entorno, son una herramienta útil para el seguimiento, monitoreo y seguridad sobre los flujos de la cadena de suministro [63, 64].

\subsection{Redes neuronales artificiales}

Las RNA son técnicas de inteligencia artificial diseñadas para resolver problemas asociados a la capacidad de aprendizaje. Un sistema inteligente que sea capaz de aprender, puede ajustarse para realizar tareas de clasificación, regresión, agrupación o ajuste, que permiten predecir, diagnósticas o recomendar. Así pues, las cadenas de suministro presentan retos interesantes para las RNA, tal es el caso de [65] que proponen un modelo de predicción de demanda del consumidor para la definición de estrategias apropiadas, empleando arquitectura de redes tipos perceptrón. Así mismo, en [66] presentan las principales fortalezas de las RNA para recomendar proveedores y uso de recursos dentro de las cadenas de suministro. Por su parte en [67] aplican las RNA para el diseño de sistemas de predicción y monitoreo que permitan la construcción de mecanismos de alerta.

Una correcta selección y clasificación de los recursos empleados en los procesos logísticos, son de vital importancia a la hora de medir el rendimiento de las cadenas de suministro, es así que en [68] plantean que las RNA son apropiadas para la selección de proveedores de acuerdo un conjunto de datos históricos de cumplimiento. Así mismo, es posible lograr una evaluación de los proveedores utilizando datos de aprendizaje que permitan una correcta toma de decisiones $[69,70,71]$ y agrupamiento de proveedores por medio de identificación de patrones [72,73].

\subsection{Sistemas de razonamiento y lógica difusa}

Las cadenas de suministro, se estructuran a partir de diversos procesos que son susceptibles a mejoramiento, optimización o manejo de información (Roy et al., 2016). Con la información es posible realizar reglas de razonamiento y ser aprovechadas por técnicas básicas como los sistemas expertos y la lógica difusa. Ejemplo de lo anterior, se tiene el trabajo realizado por [74] que riesgo al cual se somete el diseño de una nueva cadena de suministro.

La lógica difusa con su capacidad de evaluar elementos a partir de su grado pertenencia, logra que un individuo pertenezca a 1 o más conjuntos, es así como las salidas de los sistemas de control difuso se caracterizan por ser más precisas que otras técnicas de IA. Con base en lo anterior, en [75] presentan una solución que permite el razonamiento relacionado con el nivel de satisfacción del cliente. Igualmente en [76] plantean una solución para el diseño de redes de cadenas de suministro en el contexto de ambientes complejos y con incertidumbre.

La toma de decisiones no es ajena a los impactos de la lógica difusa; lo anterior se evidencia en trabajos como el de [77] donde se propone un modelo que apoya las decisiones en las negociaciones, permitiendo una mayor precisión en la evaluación de la negociación. En [78] proponen un sistema de control difuso que permite una apropiada selección de proveedores bajo incertidumbre en cadenas de suministro circular. También en [22] presentan un modelo predicción que emplea lógica difusa adapta- 
tiva para el mejoramiento del uso de recursos dentro de la cadena de suministro, como por ejemplo: el uso de agua, herramientas de construcción y materiales.

\section{OPORTUNIDADES EN LA TRANSFORMACIÓN DIGITAL DE LAS CADENAS DE SUMINISTRO}

En esta sección se analiza la información recabada; inicialmente se presentan los hallazgos desde el punto de vista de las oportunidades en las cuales se pueden destacar dentro de las cadenas de suministro. Posteriormente se presenta una discusión a la luz de las preguntas de investigación planteadas para esta investigación.

\subsection{Análisis de hallazgos}

El enfoque de la transformación de la cadena de suministro percibida desde las oportunidades y la revisión de los autores, se presenta en la siguiente Tabla 2. En esta tabla se integra la información teniendo en cuenta los requisitos actuales de las diferentes partes interesadas del negocio y los resultados del sistema relacionado.

Tabla 2 - Oportunidades para la Transformación digital de la cadena de suministro con IA

\begin{tabular}{|c|c|c|}
\hline PROCESO & TÉCNICAS IA & OPORTUNIDAD \\
\hline \multirow{2}{*}{$\begin{array}{l}\text { Gestión de Alma- } \\
\text { cenamiento }\end{array}$} & $\begin{array}{l}\text { Algoritmos } \\
\text { Genéticos }\end{array}$ & $\begin{array}{l}\text { Optimización de la capacidad de búsqueda en el almacena- } \\
\text { miento }\end{array}$ \\
\hline & $\begin{array}{l}\text { Algoritmos } \\
\text { Genéticos }\end{array}$ & $\begin{array}{l}\text { Optimización en la selección y la asignación de los operado- } \\
\text { res de despacho en ambientes de incertidumbre }\end{array}$ \\
\hline \multirow{3}{*}{$\begin{array}{l}\text { Gestión de inven- } \\
\text { tarios }\end{array}$} & $\begin{array}{l}\text { Agentes } \\
\text { inteligentes }\end{array}$ & $\begin{array}{l}\text {-Reducción de costos. } \\
\text {-Mejoramiento del promedio de nivel de servicio }\end{array}$ \\
\hline & $\begin{array}{l}\text { Algoritmos } \\
\text { Genéticos }\end{array}$ & $\begin{array}{l}\text {-Control para disponibilidad } \\
\text {-Ubicación eficiente. } \\
\text {-Modelado en la cadena de suministro. }\end{array}$ \\
\hline & $\begin{array}{l}\text { Redes } \\
\text { Neuronales }\end{array}$ & $\begin{array}{l}\text {-Integración de inventarios } \\
\text { - Rastreo de la variabilidad del proceso }\end{array}$ \\
\hline \multirow{2}{*}{$\begin{array}{l}\text { Demanda } \\
\text { /pronósticos }\end{array}$} & \multirow{2}{*}{$\begin{array}{l}\text { Agentes } \\
\text { inteligentes }\end{array}$} & Predicción de la demanda. \\
\hline & & Predicción de la demanda para reducir el efecto látigo \\
\hline \multirow{3}{*}{$\begin{array}{l}\text { Gestión de la ca- } \\
\text { dena de Suminis- } \\
\text { tro }\end{array}$} & $\begin{array}{l}\text { Aprendizaje } \\
\text { de máquina }\end{array}$ & $\begin{array}{l}\text {-Mejora proceso de toma de decisiones } \\
\text {-Análisis de datos basados en el contexto. } \\
\text {-Predicción de la demanda a partir de datos de clasificación y } \\
\text { agrupamiento }\end{array}$ \\
\hline & $\begin{array}{l}\text { Redes } \\
\text { Neuronales. }\end{array}$ & Análisis de factores de riesgo \\
\hline & $\begin{array}{l}\text { Agentes } \\
\text { inteligentes }\end{array}$ & $\begin{array}{l}\text { Integración de las cadenas de suministro. } \\
\text {-Uso de vocabularios compartidos. } \\
\text {-Minimización de los tiempos de entrega y costos de produc- } \\
\text { ción. } \\
\text {-Programación de producción. } \\
\text {-Seguimiento y suministro de información } \\
\text {-Negociación colaborativa }\end{array}$ \\
\hline $\begin{array}{l}\text { Gestión de Pro- } \\
\text { veedores }\end{array}$ & $\begin{array}{l}\text { Lógica } \\
\text { Difusa }\end{array}$ & $\begin{array}{l}\text {-Mejoramiento del uso de recursos dentro de la cadena de } \\
\text { suministro. }\end{array}$ \\
\hline
\end{tabular}




\begin{tabular}{|c|c|c|}
\hline \multicolumn{3}{|r|}{ e suministro del sector bananero basado en software con inteligencia artíficial } \\
\hline & & $\begin{array}{l}\text {-Diseño de redes en el contexto de ambientes complejos y } \\
\text { con incertidumbre. } \\
\text {-Mejoramiento en la negociación y la colaboración }\end{array}$ \\
\hline & $\begin{array}{l}\text { Redes } \\
\text { Neuronales }\end{array}$ & $\begin{array}{l}\text {-Reducción de la subjetividad en el proceso de selección de } \\
\text { proveedores } \\
\text {-Mejora en la eficiencia del trabajo }\end{array}$ \\
\hline & $\begin{array}{l}\text { Agentes } \\
\text { Inteligentes }\end{array}$ & -Mejora en el desempeño de proveedores para el retail \\
\hline & $\begin{array}{l}\text { Algoritmos } \\
\text { Genéticos }\end{array}$ & $\begin{array}{l}\text {-Optimización de los costos en la selección y evaluación de } \\
\text { proveedores }\end{array}$ \\
\hline \multirow[t]{3}{*}{$\begin{array}{l}\text { Gestión de Distri- } \\
\text { bución }\end{array}$} & $\begin{array}{l}\text { Algoritmos } \\
\text { Genéticos }\end{array}$ & $\begin{array}{l}\text { Mejorar la estabilidad de distribución mediante modelo de } \\
\text { alerta temprana de riesgo }\end{array}$ \\
\hline & $\begin{array}{l}\text { Agentes } \\
\text { Inteligentes }\end{array}$ & $\begin{array}{l}\text {-Optimización de costos mediante la colaboración, coopera- } \\
\text { ción y negociación de agentes. } \\
\text {-Minimizar los tiempos de entrega y costos de producción. }\end{array}$ \\
\hline & Lógica difusa & $\begin{array}{l}\text { Mejora el nivel de servicio del cliente mediante modelo basa- } \\
\text { do en lógica difusa para el razonamiento }\end{array}$ \\
\hline
\end{tabular}

Según lo expuesto en la Tabla 2, la IA impacta la gestión del almacenamiento, la gestión de inventarios, los pronósticos de demanda, la gestión integrada de la cadena de suministro, la gestión de proveedores y la gestión de distribución.

\subsection{Discusión de la oportunidades identificadas}

Con base en la información recabada, el análisis y consolidación de la misma, a continuación se da respuesta a cada una de las preguntas de investigación planteadas (ver sección 3.1).

Pregunta 1: ¿Cuáles son los principales impactos que genera el software con inteligencia artificial en la cadena de suministro del sector bananero en Colombia?

Respuesta: Los principales impactos que se generan en las cadenas de suministro del sector bananero y que pueden ser aprovechados por los profesionales del sector son:

Impacto 1: Optimización en la gestión de almacenamiento: La industria bananera depende en especial cuidado de los tiempos de almacenamiento. Dependiendo del contexto, tipo de producto y contexto, los cambios en los tiempos puede afectar ostensiblemente la calidad de los productos; con sistemas inteligentes basados en algoritmos genéticos o redes neuronales se puede optimizar o predecir de una manera apropiada dichos tiempos.

Impacto 2: Mejoría en la predicción de la demanda: Los sistemas inteligentes en especial las redes neuronales artificial, facilita la toma de decisiones en la comercialización de banano mejore, ya que, se pueden tener en cuenta datos de las poblaciones y clientes, con el fin de considerar cambios en el ambiente y de esta manera estar preparados.

Impacto 3: Apoyo inteligente en la gestión de inventarios: Los agentes inteligentes, las redes neuronales y los agentes genéticos, permiten la optimización en la gestión de inventarios, pues garantizan mantener los inventarios adecuados con respecto a la demanda y mejora significativa en los costos y en el nivel de servicio.

Impacto 4: Gestión de proveedores: Por medio de las redes neuronales, la lógica difusa, los agentes inteligentes y los algoritmos genéticos, se reduce la subjetividad de la evaluación de los procesos de 
selección, evaluación e identificación de proveedores, así mismo mejora la eficiencia del trabajo en ambientes con incertidumbre.

Impacto 5: Métodos inteligentes para la distribución: Las alertas tempranas de riesgo en la distribución, genera estabilidad en ambientes distributivos a partir de la cooperación, colaboración y negociación, minimizan los tiempos de entrega y los costos de transporte, mediante soluciones como lógica difusa y algoritmos inteligentes.

Pregunta 2: $¿ E$ El software con inteligencia artificial fortalece la cadena de suministro frente a cambio críticos en el entorno?

Respuesta: La inteligencia artificial ofrece grandes oportunidades para las cadenas de suministro, las cuales pueden concretarse en métodos que faciliten la identificación temprana de riesgos, adaptación, flexibilidad y sensibilidad al contexto de ejecución de las mismas. No obstante, se deben hacer avances y definir líneas de trabajo en donde se pueda tratar los siguientes aspectos:

- Definición de métodos de identificación, análisis, diseño y validación de flujos de las cadenas de suministro, donde se tenga en cuenta información del entorno y el contexto de ejecución.

- Diseño de protocolos de comunicación e intercambio de información y conocimiento que permitan una integración entre las cadenas de suministro.

- Diseñar procesos logísticos que permitan la recolección, procesamiento, almacenamiento y distribución de información que faciliten el aprendizaje a partir de datos para la construcción de sistemas de predicción y recomendación.

- Construcción de ontologías de dominio del sector bananero que faciliten el intercambio de conocimiento y a su vez apoyen la construcción de mecanismos de razonamiento inteligente basados en semántica.

\section{CONCLUSIONES}

Optimización en la gestión de la cadena de suministro, se convierte en una ventaja competitiva, que el software basado en técnicas de la IA ofrece. La IA apoya las decisiones humanas y potencializa los resultados rápidamente requeridos ante los cambios de la demanda y del entorno, es así cómo se evalúan los factores de riesgo en la cadena de suministro, mejora en la comunicación, mejora la predicción de los pronósticos, y mejora la selección y evaluación de proveedores bajo alta incertidumbre.

Esta revisión de literatura identifica los principales aportes de la IA en la cadena de suministro, a partir de ello se hace un análisis de los impactos reflejados en oportunidades para el sector bananero en Colombia. Dichos impactos se concentran en oportunidades en los siguientes verbos: predicción, recomendación, selección y optimización.

En especial un área que tiene gran potencial es la representación basada en el contexto y semántica, pues las investigaciones como los agentes inteligentes los permiten. Dicha representación facilitará la integración de distintas cadenas de suministro, donde sea posible apoyarse e intercambiar información que conlleve a una flexibilidad y adaptación a los cambios del mundo de hoy.

\section{AGRADECIMIENTOS}

El conocimiento sobre software de inteligencia artificial y conceptos informáticos incluidos en este artículo debe ser reconocido gracias al apoyo del Doctor en Ingeniería - Sistemas e Informática, Dr. Jorge Eliecer Giraldo Plaza, gracias por el apoyo brindado con su conocimiento experto, sus investigaciones con respecto a este artículo y su disposición constante por participar en la escritura científica. 


\section{REFERENCIAS BIBLIOGRAFICAS}

[1] Dellino, G., Laudadio, T., Mari, R., Mastronardi, N., \& Meloni, C. (2018). A reliable decision support system for fresh food supply chain management. International Journal of Production Research, 56(4), 1458-1485. https://doi.org/10.1080/00207543.2017.1367106

[2] Muñoz-Pinzón, D. S., Polo-Roa, A., Sierra-Mantilla, E. J., \& Rueda-Uribe, D. (2020). Modelación matemática en estudio de agro-cadenas: una revisión de literatura. REVISTA POLITÉCNICA, 16(31), 110-137. https://doi.org/10.33571/rpolitec.v16n31a9

[3] Nasiri, M., Ukko, J., Saunila, M., \& Rantala, T. (2020). Managing the digital supply chain: The role of smart technologies. Technovation, 102121. https://doi.org/10.1016/j.technovation.2020.102121

[4] Wu, L., Yue, X., Jin, A., \& Yen, D. C. (2016). Smart supply chain management: a review and implications for future research. The International Journal of Logistics Management. ISSN: 0957-4093

[5] Zhao, J., Ji, M., \& Feng, B. (2020). Smarter supply chain: a literature review and practices. Journal of Data, Information and Management, 1-16. https://doi.org/10.1007/s42488-020-00025-z

[6] Büyüközkan, G., \& Göçer, F. (2018). Digital Supply Chain: Literature review and a proposed framework for future research. Computers in Industry, 97, 157-177. https://doi.org/10.1016/j.compind.2018.02.010

[7] Augura (2019). Sector Bananero en Colombia. Obtenido de Sector Bananero en Colombia: Recuperado de https://sac.org.co/sector-bananero-colombiano-crecio-en-2018/

[8] Ehie, I., \& Ferreira, L. M. D. (2019). Conceptual Development of Supply Chain Digitalization Framework. IFAC-PapersOnLine, 52(13), 2338-2342. https://doi.org/10.1016/j.ifacol.2019.11.555

[9] MinAgricultura. (2018). Cadena de Banano Indicadores e Instrumentos 2018. Recuperado de https://www.minagricultura.gov.co/paginas/default.aspx

[10] Giarratano, J., \& Riley, G. (2001). Sistemas expertos: principios y programación. Thomson.

[11] Cruz, P. P. (2011). Inteligencia artificial con aplicaciones a la ingeniería. Alfaomega.

[12] Plinere, D., \& Merkurvev, Y. (2019, November). Designing A Multi-Agent System For Improving Supply Chain Performance. In 2019 IEEE 7th IEEE Workshop on Advances in Information, Electronic and Electrical Engineering (AIEEE) (pp. 1-7). IEEE.

[13] Shalev-Shwartz, S., \& Ben-David, S. (2014). Understanding machine learning: From theory to algorithms. Cambridge university press.

[14] Wooldridge, M. (2009). An introduction to multiagent systems. John Wiley \& Sons.

[15] Korpela, K., Hallikas, J., \& Dahlberg, T. (2017, January). Digital supply chain transformation toward blockchain integration. In proceedings of the 50th Hawaii international conference on system sciences. http://hdl.handle.net/10125/41666

[16] Kamble, S. S., Gunasekaran, A., \& Gawankar, S. A. (2020). Achieving sustainable performance in a data-driven agriculture supply chain: A review for research and applications. International Journal of Production Economics, 219, 179-194. https://doi.org/10.1016/j.jpe.2019.05.022

[17] Lezoche, M., Hernandez, J. E., Díaz, M. D. M. E. A., Panetto, H., \& Kacprzyk, J. (2020). Agri-food 4.0: a survey of the supply chains and technologies for the future agriculture. Computers in Industry, 117, 103187. https://doi.org/10.1016/j.compind.2020.103187 
[18] Yinyun, L. (2015, August). Enterprise Logistics Cost Research Based on Optimized Supply Chain Model. In 2015 Sixth International Conference on Intelligent Systems Design and Engineering Applications (ISDEA) (pp. 380-383). IEEE. DOI: 10.1109 / ISDEA.2015.101

[19] Zhang, Y., Liu, S., \& Zhang, X. (2017). An optimized supply chain network model based on modified genetic algorithm. Chinese Journal of Electronics, 26(3), 468-476. DOI: 10.1049 / cje.2017.03.018

[20] Yang, H., Chung, J. K., Chen, Y., Pan, Y., Mei, Z., \& Sun, X. (2018). Ordering Strategy Analysis of Prefabricated Component Manufacturer in Construction Supply Chain. Mathematical Problems in Engineering, 2018. https://doi.org/10.1155/2018/4062871.

[21] Zhang, Y., Jiang, Y., Zhong, M., Geng, N., \& Chen, D. (2016). Robust optimization on regional WCO-for-Biodiesel supply chain under supply and demand uncertainties. Scientific Programming, 2016. https://doi.org/10.1155/2016/1087845

[22] Akinade, O. O., \& Oyedele, L. O. (2019). Integrating construction supply chains within a circular economy: An ANFIS-based waste analytics system (A-WAS). Journal of Cleaner Production, 229, 863873. https://doi.org/10.1016/j.jclepro.2019.04.232

[23] Guo, F., Liu, Q., Liu, D., \& Guo, Z. (2017). On production and green transportation coordination in a sustainable global supply chain. Sustainability, 9(11), 2071. https://doi.org/10.3390/su9112071

[24] Kusolpuchong, S., Chusap, K., Alhawari, O., \& Suer, G. (2019). A Genetic Algorithm Approach for Multi Objective Cross Dock Scheduling in Supply Chains. Procedia Manufacturing, 39, 1139-1148. https://doi.org/10.1016/j.promfg.2020.01.356

[25] Bank, M., Mazdeh, M., \& Heydari, M. (2020). Applying meta-heuristic algorithms for an integrated production-distribution problem in a two level supply chain. Uncertain Supply Chain Management, 8(1), 77-92. doi: 10.5267/j.uscm.2019.8.004

[26] Hamontree, C., Prompakdee, S., \& Koiwanit, J. (2019, October). Resource Scheduling Problem in Distribution Center. In IOP Conference Series: Materials Science and Engineering (Vol. 639, No. 1, p. 012017). IOP Publishing.

[27] Ahmadizar, F., Zeynivand, M., \& Arkat, J. (2015). Two-level vehicle routing with cross-docking in a three-echelon supply chain: A genetic algorithm approach. Applied Mathematical Modelling, 39(22), 7065-7081. https://doi.org/10.1016/j.apm.2015.03.005

[28] Boru, A., Dosdoğru, A. T., Göçken, M., \& Erol, R. (2019). A Novel Hybrid Artificial Intelligence Based Methodology for the Inventory Routing Problem. Symmetry, 11(5), 717. https://doi.org/10.3390/sym11050717

[29] Rabbani, M., Navazi, F., Farrokhi-Asl, H., \& Balali, M. (2018). A sustainable transportationlocation-routing problem with soft time windows for distribution systems. Uncertain Supply Chain Management, 6(3), 229-254. DOI: 10.5267 / j.uscm.2017.12.002

[30] Moncayo-Martínez, L. A. (2017). Supply chain design using a modified IWD algorithm. Revista Facultad de Ingeniería Universidad de Antioquia, (84), 9-16. DOI: 10.17533/udea.redin.n84a02

[31] Gong, G., Deng, Q., Gong, X., Zhang, L., Wang, H., \& Xie, H. (2018). A Bee Evolutionary Algorithm for Multiobjective Vehicle Routing Problem with Simultaneous Pickup and Delivery. Mathematical Problems in Engineering, 2018. https://doi.org/10.1155/2018/2571380

[32] Rahman, A., Shahruddin, N. S., \& Ishak, I. (2019, November). Solving the Goods Transportation Problem Using Genetic Algorithm with Nearest-Node Pairing Crossover Operator. In Journal of Physics: Conference Series (Vol. 1366, No. 1, p. 012073). IOP Publishing. DOI:10.1088/1742$6596 / 1366 / 1 / 012073$ 
[33] Fitriana, R., Moengin, P., \& Kusumaningrum, U. (2019, May). Improvement Route for Distribution Solutions MDVRP (Multi Depot Vehicle Routing Problem) using Genetic Algorithm. In IOP Conference Series: Materials Science and Engineering (Vol. 528, No. 1, p. 012042). IOP Publishing. DOI:10.1088/1757-899X/528/1/012042

[34] Frazzon, E. M., Albrecht, A., Pires, M., Israel, E., Kück, M., \& Freitag, M. (2018). Hybrid approach for the integrated scheduling of production and transport processes along supply chains. International Journal of Production Research, 56(5), 2019-2035. https://doi.org/10.1080/00207543.2017.1355118

[35] Zhou, L., Wang, X., Ni, L., \& Lin, Y. (2016). Location-routing problem with simultaneous home delivery and customer's pickup for city distribution of online shopping purchases. Sustainability, 8(8), 828. https://doi.org/10.3390/su8080828

[36] Gooran, A., Rafiei, H., \& Rabani, M. (2018). Modeling risk and uncertainty in designing reverse logistics problem. Decision Science Letters, 7(1), 13-24. DOI: 10.5267 / j.dsl.2017.5.001

[37] Soleimani, H., \& Kannan, G. (2015). A hybrid particle swarm optimization and genetic algorithm for closed-loop supply chain network design in large-scale networks. Applied Mathematical Modelling, 39(14), 3990-4012. https://doi.org/10.1016/j.apm.2014.12.016

[38] Zhou, Y., Chan, C. K., Wong, K. H., \& Lee, Y. C. E. (2015). Intelligent optimization algorithms: a stochastic closed-loop supply chain network problem involving oligopolistic competition for multiproducts and their product flow routings. Mathematical Problems in Engineering, 2015. https://doi.org/10.1155/2015/918705

[39] Afrouzy, Z. A., Paydar, M. M., Nasseri, S. H., \& Mahdavi, I. (2018). A meta-heuristic approach supported by NSGA-II for the design and plan of supply chain networks considering new product development. Journal of Industrial Engineering International, 14(1), 95-109. https://doi.org/10.1007/s40092-017-0209-7

[40] Yun, Y., Chuluunsukh, A., \& Gen, M. (2020). Sustainable Closed-Loop Supply Chain Design Problem: A Hybrid Genetic Algorithm Approach. Mathematics, 8(1), 84. https://doi.org/10.3390/math8010084

[41] Huang, R. H., Yu, T. H., \& Lee, C. Y. (2018). Rolling Supply Chain Scheduling considering Suppliers, Production, and Delivery Lot-Size. Mathematical Problems in Engineering, 2018. https://doi.org/10.1155/2018/8601209

[42] Huang, M., Yi, P., Guo, L., \& Shi, T. (2016). A modal interval based genetic algorithm for closedloop supply chain network design under uncertainty. IFAC-PapersOnLine, 49(12), 616-621. https://doi.org/10.1016/j.ifacol.2016.07.743

[43] Mohammadi, M., Tavakkoli-Moghaddam, R., Siadat, A., \& Dantan, J. Y. (2016). Design of a reliable logistics network with hub disruption under uncertainty. Applied Mathematical Modelling, 40(9-10), 5621-5642. https://doi.org/10.1016/j.apm.2016.01.011

[44] Kumar, R. S., Choudhary, A., Babu, S. A. I., Kumar, S. K., Goswami, A., \& Tiwari, M. K. (2017). Designing multi-period supply chain network considering risk and emission: A multi-objective approach. Annals of Operations Research, 250(2), 427-461. DOI 10.1007/s10479-015-2086-z

[45] Xu, Y. P., \& Liu, X. (2015, December). A New Genetic Type Method with Integrated Gradient Based Algorithm Method for Storage Optimization of Supply Chain. In 2015 International Conference on Computational Intelligence and Communication Networks (CICN) (pp. 724-726). IEEE. DOI: 10.1109 / CICN.2015.147

[46] Dabibi, M., Moghaddam, B., \& Kazemi, M. (2016). Locating distribution/service centers based on multi objective decision making using set covering and proximity to stock market. International Journal of Industrial Engineering Computations, 7(4), 635-648. DOI: 10.5267/j.jijec.2016.3.002 
[47] Wang, Y., Yuan, Y., Assogba, K., Gong, K., Wang, H., Xu, M., \& Wang, Y. (2018). Design and Profit Allocation in Two-Echelon Heterogeneous Cooperative Logistics Network Optimization. Journal of Advanced Transportation, 2018. https://doi.org/10.1155/2018/4607493

[48] Saif-Eddine, A. S., El-Beheiry, M. M., \& El-Kharbotly, A. K. (2019). An improved genetic algorithm for optimizing total supply chain cost in inventory location routing problem. Ain Shams Engineering Journal, 10(1), 63-76. https://doi.org/10.1016/j.asej.2018.09.002

[49]Wang, Y., Geng, X., Zhang, F., \& Ruan, J. (2018). An immune genetic algorithm for multi-echelon inventory cost control of IOT based supply chains. IEEE Access, 6, 8547-8555. DOI: 10.1109/ACCESS.2018.2799306

[50] Jahani, H., Alavifard, F., Ivanov, D., \& Ghasemishabankareh, B. (2019). Managing the risk of supply chain bankruptcy in supply chain network redesign. IFAC-PapersOnLine, 52(13), 2431-2436. https://doi.org/10.1016/j.ifacol.2019.11.571

[51] Shen, L., Li, F., Li, C., Wang, Y., Qian, X., Feng, T., \& Wang, C. (2020). Inventory optimization of fresh agricultural products supply chain based on agricultural superdocking. Journal of Advanced Transportation, 2020. https://doi.org/10.1155/2020/2724164

[52] Simić, D., Svirčević, V., \& Simić, S. (2015). A hybrid evolutionary model for supplier assessment and selection in inbound logistics. Journal of Applied Logic, 13(2), 138-147. IFAC PapersOnLine 52-13 (2019) 2431-2436

[53] Wang, H. S., Tu, C. H., \& Chen, K. H. (2015). Supplier selection and production planning by using guided genetic algorithm and dynamic nondominated sorting genetic algorithm II. https://doi.org/10.1155/2015/260205

[54] Mahmud, S., Rahman, M., Hasan, M., \& Hossain, M. (2016). Minimizing the bullwhip effect in a single product multistage supply chain using genetic algorithm. Uncertain Supply Chain Management, 4(2), 137-146. DOI: $10.5267 /$ j.uscm.2015.11.001

[55] Nakhjirkan, S., \& Mokhatab Rafiei, F. (2017). An integrated multi-echelon supply chain network design considering stochastic demand: a genetic algorithm based solution. PrometTraffic\&Transportation, 29(4), 391-400. https://doi.org/10.7307/ptt.v29i4.2193

[56] Jing, Y., \& Li, W. (2018). Integrated recycling-integrated production-distribution planning for decentralized closed-loop supply chain. Journal of Industrial \& Management Optimization, 14(2), 511-539. doi: $10.3934 / j i m o .2017058$

[57]Agrawal, A. K., \& Yadav, S. (2020). Price and profit structuring for single manufacturer multi-buyer integrated inventory supply chain under price-sensitive demand condition. Computers \& Industrial Engineering, 139. https://doi.org/10.1016/j.cie.2019.106208

[58] Gamasaee, R., Zarandi, M. F., \& Turksen, I. B. (2015, August). A type-2 fuzzy intelligent agent based on sparse kernel machines for reducing bullwhip effect in supply chain. In 2015 Annual Conference of the North American Fuzzy Information Processing Society (NAFIPS) held jointly with 2015 5th World Conference on Soft Computing (WConSC) (pp. 1-7). IEEE. DOI: 10.1109/NAFIPSWConSC.2015.7284207

[59] Gonçalo, T. E. E., \& Morais, D. C. (2015, October). Agent-based negotiation protocol for selecting transportation providers in a retail company. In 2015 IEEE International Conference on Systems, Man, and Cybernetics (pp. 263-267). IEEE. DOI: 10.1109/SMC.2015.58

[60] Hsieh, F. S. (2015, November). Scheduling sustainable supply chains based on multi-agent systems and workflow models. In 2015 10th International Conference on Intelligent Systems and Knowledge Engineering (ISKE) (pp. 252-259). IEEE. DOI: 10.1109/ISKE.2015.20 
Oportunidades para la transformación digital de la cadena de suministro del sector bananero basado en software con inteligencia artíficial

[61] Saoud, A., \& Bellabdaoui, A. (2017, April). Model of distributed hierarchical framework for carrier collaboration. In 2017 International Colloquium on Logistics and Supply Chain Management (LOGISTIQUA) (pp. 160-165). IEEE. DOI: 10.1109/LOGISTIQUA.2018.8428266

[62] Fu, D., Zhang, H. T., Dutta, A., \& Chen, G. (2019). A Cooperative Distributed Model Predictive Control Approach to Supply Chain Management. IEEE Transactions on Systems, Man, and Cybernetics: Systems. DOI: 10.1109/TSMC.2019.2930714

[63] Fang, D., \& Puqing, W. (2015, August). Simulating the Structural Evolution in Agri-food Supply Chain: An Agent-Based Model. In 2015 7th International Conference on Intelligent Human-Machine Systems and Cybernetics (Vol. 1, pp. 214-219). IEEE. DOI: 10.1109/IHMSC.2015.254

[64] Du, J., Sugumaran, V., \& Gao, B. (2017). RFID and multi-agent based architecture for information sharing in prefabricated component supply chain. IEEE Access, 5, 4132-4139. DOI: 10.1109/ACCESS.2017.2665778

[65] Slimani, I., El Farissi, I., \& Achchab, S. (2015, December). Artificial neural networks for demand forecasting: Application using Moroccan supermarket data. In 2015 15th International Conference on Intelligent Systems Design and Applications (ISDA) (pp. 266-271). IEEE. DOI: 10.1109/ISDA.2015.7489236

[66] Bousqaoui, H., Achchab, S., \& Tikito, K. (2017, October). Machine learning applications in supply chains: An emphasis on neural network applications. In 2017 3rd International Conference of Cloud Computing Technologies and Applications (CloudTech) (pp. 1-7). IEEE. DOI: 10.1109/CloudTech.2017.8284722

[67] Li, Z., Li, G., Zhang, Y., Chen, J., \& Dai, Y. (2019, October). Risk Early Warning Model for Distribution Network Material Supply Chain of Electric Power Enterprises. In 2019 12th International Conference on Intelligent Computation Technology and Automation (ICICTA) (pp. 700-707). IEEE. DOI: 10.1109/ICICTA49267.2019.00154

[68] Yangyang, Z., Zhonghua, C., Quanyue, M., \& Qian, W. (2019, October). Research on Supplier Selection Method Based on BP Neural Network. In 2019 IEEE 1st International Conference on Civil Aviation Safety and Information Technology (ICCASIT) (pp. 344-347). IEEE.

[69] Lin, T. Y., Chuang, H. H. C., \& Yu, F. (2018, August). Tracking Supply Chain Process Variability with Unsupervised Cluster Traversal. In 2018 IEEE 16th Intl Conf on Dependable, Autonomic and Secure Computing, 16th Intl Conf on Pervasive Intelligence and Computing, 4th Intl Conf on Big Data Intelligence and Computing and Cyber Science and Technology Congress (DASC/PiCom/DataCom/CyberSciTech) (pp. 966-973). IEEE. DOI: 10.1109/DASC/PiCom/DataCom/CyberSciTec.2018.000-2

[70] Xuefeng, H., Chi, Z., Yuewu, J., \& Xingzheng, A. (2019, July). Risk Evaluation of Agricultural Product Supply Chain Based on BP Neural Network. In 2019 16th International Conference on Service Systems and Service Management (ICSSSM) (pp. 1-8). IEEE. DOI: 10.1109/ICSSSM.2019.8887744

[71] Cheng, Y., Peng, J., Gu, X., Zhang, X., Liu, W., Zhou, Z., ... \& Huang, Z. (2020). An intelligent supplier evaluation model based on data-driven support vector regression in global supply chain. Computers \& Industrial Engineering, 139, 105834. https://doi.org/10.1016/j.cie.2019.04.047

[72] Liu, P., \& Yi, S. (2016). New Algorithm for Evaluating the Green Supply Chain Performance in an Uncertain Environment. Sustainability, 8(10), 960. https://doi.org/10.3390/su8100960

[73] Pereira, M. M., \& Frazzon, E. M. (2019). Towards a Predictive Approach for Omni-channel Retailing Supply Chains. IFAC-PapersOnLine, 52(13), 844-850. https://doi.org/10.1016/j.ifacol.2019.11.235 
[74] Kara, M. E., Fırat, S. Ü. O., \& Ghadge, A. (2018). A data mining-based framework for supply chain risk management. Computers \& Industrial Engineering, 105570. https://doi.org/10.1016/j.cie.2018.12.017

[75] Besheli, S. F., Keshteli, R. N., Emami, S., \& Rasouli, S. M. (2017). A fuzzy dynamic multi-objective multi-item model by considering customer satisfaction in a supply chain. Scientia Iranica. Transaction E, Industrial Engineering, 24(5), 2623-2639. Doi: 10.24200/SCI.2017.439

[76] Ammar, O. B., Marian, H., \& Dolgui, A. (2015, May). Supply planning for multi-levels assembly system under random lead times. In 15th IFAC Symposium on Information Control Problems in Manufacturing-INCOM 2015 (Vol. 48, No. 3, pp. Pages-254). Elsevier Science, IFACPapersOnline. net. https://doi.org/10.1016/j.ifacol.2015.06.090

[77] Yahia, W. B., Ayadi, O., \& Masmoudi, F. (2017). A fuzzy-based negotiation approach for collaborative planning in manufacturing supply chains. Journal of Intelligent Manufacturing, 28(8), 1987-2006.

[78] Govindan, K., Mina, H., Esmaeili, A., \& Gholami-Zanjani, S. M. (2020). An integrated hybrid approach for circular supplier selection and closed loop supply chain network design under uncertainty. Journal of Cleaner Production, 242, 118317. https://doi.org/10.1016/j.jclepro.2019.118317 